\title{
A New Parameterization of an Asymmetry Factor of Cirrus Clouds for Climate Models
}

\author{
QIANG FU \\ Department of Atmospheric Sciences, University of Washington, Seattle, Washington
}

(Manuscript received 6 September 2006, in final form 4 January 2007)

\begin{abstract}
The aspect ratio (AR) of a nonspherical ice particle is identified as the key microphysical parameter to determine its asymmetry factor for solar radiation. The mean effective AR is defined for cirrus clouds containing various nonspherical ice particles. A new parameterization of the asymmetry factor of cirrus clouds in terms of AR and mean effective size, $D_{g e}$, is developed for solar radiation. It is based on geometric ray-tracing calculations for hexagonal ice crystals with a simple representation of particle surface roughness. The present parameterization well reproduces the asymmetry factors of complicated ice particles such as bullet rosettes, aggregates with rough surfaces, and fractal crystals and agrees well with observations. It thus can be properly applied to cirrus clouds containing various nonspherical ice particles. The asymmetry factor from this parameterization in the visible spectrum ranges from about 0.73 to more than 0.85 .

Radiative transfer calculations show that for a cirrus cloud with an optical depth of 4 and a solar zenith angle of $60^{\circ}$, changes in AR from 1.0 to 0.5 or from 1.0 to 0.1 result in differences in reflected solar fluxes of about -30 or $-70 \mathrm{~W} \mathrm{~m}^{-2}$, respectively. For the same cloudy conditions, the effect of ice particle surface roughness on the reflected solar flux is found to be about $20 \mathrm{~W} \mathrm{~m}^{-2}$.
\end{abstract}

\section{Introduction}

A parameterization of the solar radiative properties of cirrus clouds was developed by Fu (1996) based on light scattering calculations for hexagonal ice particles. Using ice water content (IWC) and generalized effective size $\left(D_{\text {ge }}\right)$, which conserve both volume and projected area of nonspherical ice particles, the parameterization for the extinction coefficient and singlescattering albedo can be properly applied to cirrus clouds containing various nonspherical particles such as plates, columns, bullet rosettes, and aggregates ( Fu 1996; Grenfell and Warren 1999; Edwards et al. 2007). However, the parameterization of the asymmetry factor in terms of $D_{\mathrm{ge}}$ is still sensitive to the habit and shapes of ice crystals assumed in the light scattering calculations (Fu 1996; Edwards et al. 2007).

The asymmetry factor, $g$, is the first moment of the scattering phase function. The quantities $(1-g) / 2$ and $(1+g) / 2$ can be interpreted as the integrated fraction of energy backscattered and forward scattered, respec-

Corresponding author address: Dr. Qiang Fu, Department of Atmospheric Sciences, University of Washington, Box 351640, Seattle, WA 98195.

E-mail: qfu@atmos.washington.edu tively. The global albedo of clouds in the visible, based on a two-stream radiative transfer equation (Liou 2002), is

$$
R=\frac{\sqrt{3}(1-g) \tau}{2+\sqrt{3}(1-g) \tau}
$$

where $\tau$ is the cloud optical depth. The change in the albedo, $\Delta R$, due to a change in the asymmetry factor, $\Delta g$, can then be derived in the following form:

$$
\Delta R=-\frac{2}{1-g} \times \frac{R}{2+\sqrt{3}(1-g) \tau} \Delta g .
$$

Thus, for a typical cirrus asymmetry factor of 0.8 and $\Delta g$ of \pm 0.05 , the differences in albedo are about $\mp 0.03$, $\mp 0.06$, and $\mp 0.06$ for the optical depth of 1,4 , and 10 , respectively. Modeling and prediction of climate therefore require that the parameterization of the asymmetry factor of cirrus clouds be as accurate as possible.

The asymmetry factors of cirrus clouds derived from observations are 0.7 from Stephens et al. (1990), 0.75 from Garrett et al. (2001), and 0.8 from Francis et al. (1994). Theoretical calculations of asymmetry factors for ice particles in the solar radiation are usually based on the geometric ray tracing technique (e.g., Takano and Liou 1989; Macke 1993; Yang and Liou 1995). Dif-

DOI: $10.1175 / 2007 J A S 2289.1$ 


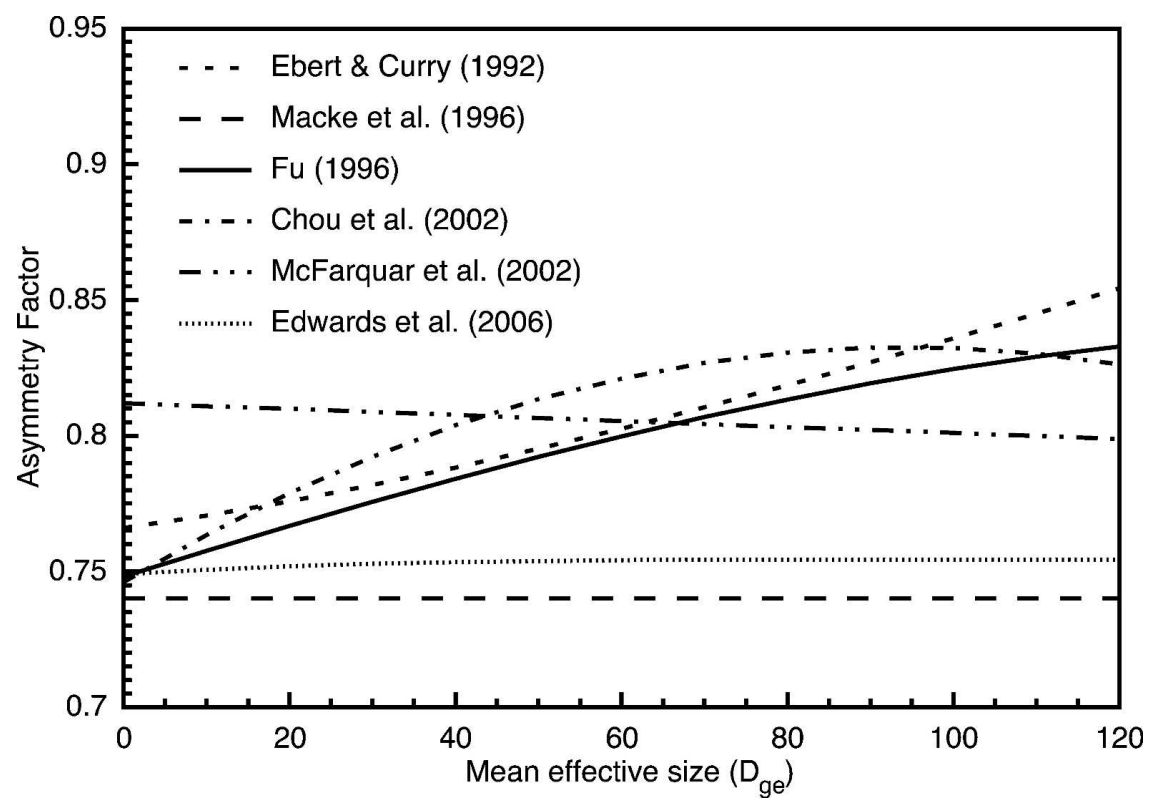

FIG. 1. Parameterizations of asymmetry factor of cirrus clouds in terms of the mean effective ice particle size from different studies for the visible radiation. Here all parameterizations are converted in terms of $D_{\mathrm{ge}}$ as defined in $\mathrm{Fu}$ (1996) for comparison purposes.

ferent parameterizations of asymmetry factors in terms of mean effective size of ice particles have been developed and used in climate models (e.g., Ebert and Curry 1992; Fu 1996; Macke et al. 1996; Chou et al. 2002; McFarquhar et al. 2002; Edwards et al. 2007). Shown in Fig. 1 are the asymmetry factors in the visible spectrum from these parameterizations, which assumed different habits and shapes and ice particle size distributions. We see different dependences of $g$ on $D_{\text {ge }}$, and for a given $D_{\text {ge }}$ the asymmetry factor ranges from 0.74 to more than 0.81. This indicates that the mean effective size may not be an appropriate microphysical parameter being used for the parameterization of the asymmetry factor. In this study, we identify a different microphysical parameter, the aspect ratio (AR) of ice particles, to determine the asymmetry factor in the solar radiation. We show that $g$ also depends on $D_{\text {ge }}$ in the near-IR when the absorption becomes significant.

In section 2 we define a mean effective AR to represent its impact on the asymmetry factor of cirrus clouds. In section 3 we develop a new parameterization of the asymmetry factor in terms of the effective aspect ratio and $D_{\text {ge }}$ based on light scattering by hexagonal ice particles. The new parameterization is tested in section 4 to show that it can be properly applied to complicated ice particle shapes. More discussions on this parameterization are given in section 5. Section 6 presents the effect of mean effective aspect ratio and ice particle surface roughness on the cirrus radiative en- ergy budget. A summary and conclusions are given in section 7 .

\section{Definition of mean effective aspect ratio}

The asymmetry factor of hexagonal ice particles in the solar spectrum is dominated by its aspect ratio, $D / L$, where $D$ and $L$ are the particle width and length, respectively. Figure 2 shows the $g$ of hexagonal ice crystals and delta-transmission fraction associated with light beams passing through the parallel planes of the ice crystals (Takano and Liou 1989) versus $D / L$ based on the geometric optics method at a wavelength of 0.56 $\mu \mathrm{m}$. We see that the asymmetry factor is very sensitive to the particle aspect ratio, ranging from 0.77 to above 0.9 with the minimum value when $D / L$ is about one.

Figure 3 shows the asymmetry factor and the deltatransmission fraction versus ice particle length $L$ for a $D / L$ of 0.5 at wavelengths of 0.56 and $1.50 \mu \mathrm{m}$. In the visible, both quantities are independent of the ice particle size for given $D / L$. This is actually true for all wavelengths shorter than about $1.4 \mu \mathrm{m}$ (about $85 \%$ of solar energy is contained in this interval) where the ice absorption is small (Fu 1996). Dependence of the asymmetry factor on the $D_{\mathrm{ge}}$ in the visible, as shown in Fig. 1 , can be largely explained by the dependence of the aspect ratio on the ice particle size assumed in the parameterization.

For hexagonal ice particles in the visible, the asym- 

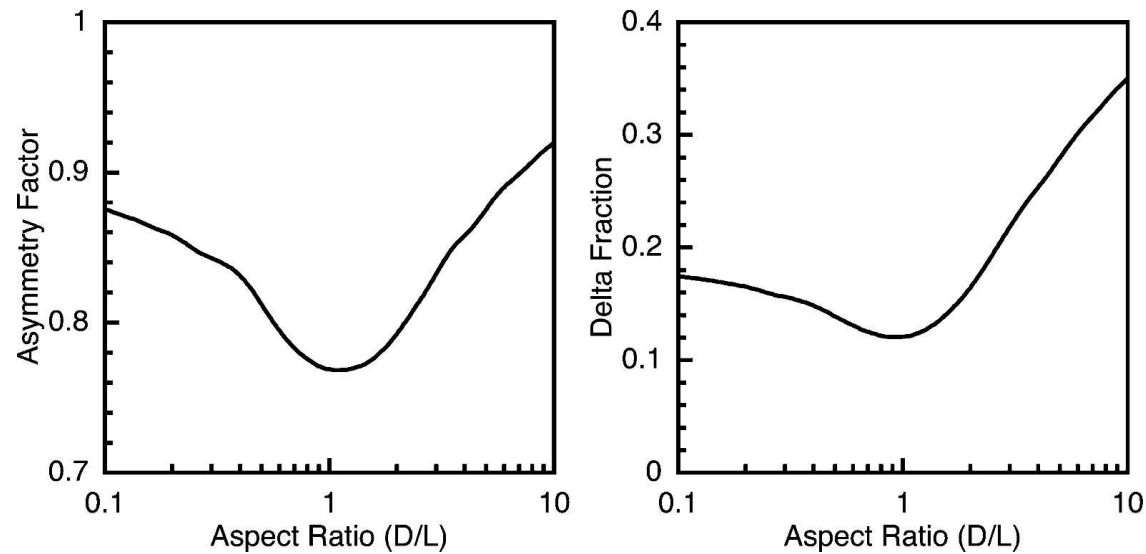

FIG. 2. Asymmetry factor and delta-transmission fraction of hexagonal ice crystals as functions of the aspect ratio $(D / L)$ at the wavelength of $0.56 \mu \mathrm{m}$ derived using the geometric ray-tracing method.

metry factor is uniquely determined by the aspect ratio (see Fig. 2), but the aspect ratio (thus the asymmetry factor) is not uniquely determined by the ice particle size. Thus it is more suitable to parameterize the asymmetry factor of nonspherical ice particles in terms of aspect ratio instead of $D_{\mathrm{ge}}$. Here we define the mean effective aspect ratio for given ice particle size distributions in the form

$$
\mathrm{AR}=\frac{\int_{L_{\min }}^{L_{\max }}(D / L) A_{c} n(L) d L}{\int_{L_{\min }}^{L_{\max }} A_{c} n(L) d L},
$$

where $D$ and $L$ are the width and length of the hexagonal component, $A_{c}$ is its projected area, and $n(L)$ is the ice crystal size distribution. The mean aspect ratio can also be defined for more complicated shapes such as bullet rosettes and aggregates by considering their individual components.

Asymmetry factors for more complicated shapes, such as hollow columns, bullet rosettes, and aggregates (Takano and Liou 1995; Yang and Liou 1998), are within the same range shown in Fig. 2, and these particle types essentially behave like their single components (Iaquinta et al. 1995; Macke et al. 1996). Thus, the parameterization of the asymmetry factor in terms of AR based on light scattering by hexagonal ice particles is expected to be generally applied to cirrus clouds containing various nonspherical ice particles.

The natural ice particles in cirrus clouds may have rough surfaces, which should also be taken into account
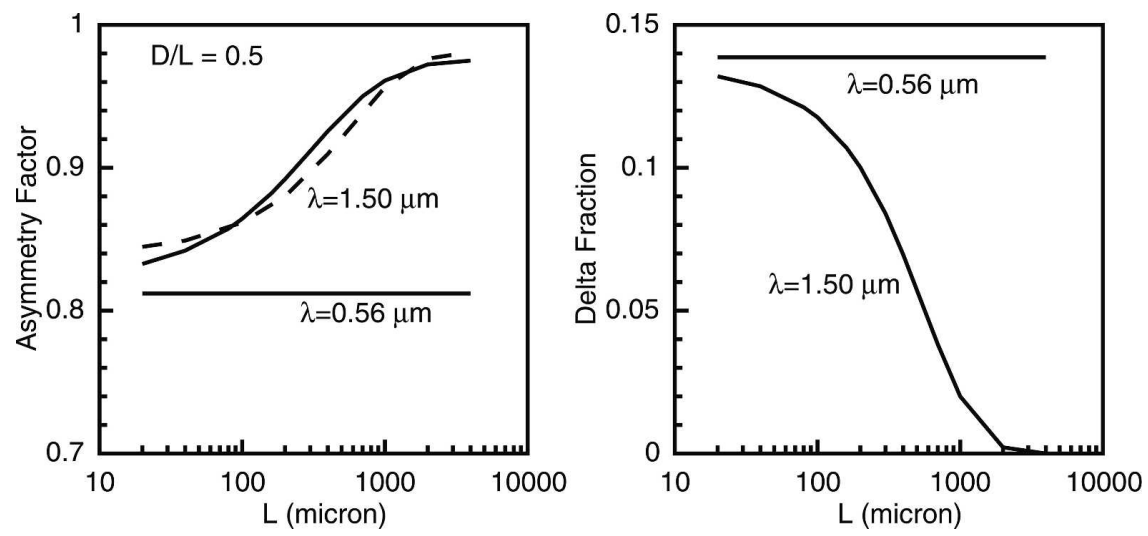

FIG. 3. Asymmetry factor and delta-transmission fraction for hexagonal ice crystals using an aspect ratio of 0.5 vs ice crystal length, $L(\mu \mathrm{m})$, at wavelengths of 0.56 and $1.50 \mu \mathrm{m}$. The dashed line for the asymmetry factor at the wavelength of $1.50 \mu \mathrm{m}$ is derived from $(1 / 2 \tilde{\omega})+$ $[1-(1 / 2 \tilde{\omega})] \times 0.68$ [see Eq. (2.2)], where $\tilde{\omega}$ decreases from 0.973 to 0.532 as $L$ increases from 20 to $4000 \mu \mathrm{m}$, and 0.68 is the averaged $g^{\prime}$. 
(e.g., Macke et al. 1996; Yang and Liou 1998). The ice particles with rough surfaces should not have the delta transmission associated with light beams passing through the parallel planes of ice crystals. Herein we simply represent the effect of ice particle surface roughness on $g$ by removing the contribution of the delta transmission to $g$ and assuming that the removed part has the same asymmetry factor as the remaining part.

For wavelengths longer than $1.4 \mu \mathrm{m}$, the asymmetry factor may depend not only on $D / L$ but also on the ice particle size. Figure 3 shows that at $1.5 \mu \mathrm{m}$, where ice exhibits an absorption peak, the asymmetry factor increases with the ice particle size for a given $D / L$. This phenomenon can be explained as follows. In the geometric optics limit, the scattering phase function is composed of the diffraction peak and geometric reflection and refraction including the delta transmission. The fraction of diffracted rays is $1 / 2 \tilde{\omega}$ (Fu 1996) where $\tilde{\omega}$ is the single-scattering albedo. Since the diffracted rays are largely confined in the forward direction, their asymmetry factor can be approximated as one. Thus the total asymmetry factor, $g$, can be generally expressed as

$$
g=\frac{1}{2 \tilde{\omega}}+\left(1-\frac{1}{2 \tilde{\omega}}\right) g^{\prime}=\frac{1-g^{\prime}}{2 \tilde{\omega}}+g^{\prime},
$$

where $g^{\prime}$ is the asymmetry factor associated with the geometric reflection and refraction. At wavelengths with strong absorption, as the single-scattering albedo decreases with the increase of ice particle size (Fu 1996), the asymmetry factor increases following Eq. (2.2). We derive the asymmetry factor at the wavelength of $1.5 \mu \mathrm{m}$ based on Eq. (2.2) using a constant $g^{\prime}$ of 0.68 , which is shown as a dashed line in Fig. 3. It indicates that the dependence of $g$ on $L$ is largely through the dependence of $\tilde{\omega}$ on $L$ and the dependence of $g^{\prime}$ on $L$ has small impact on $g$. Note that $1 / 2 \tilde{\omega}$ is 0.50 for wavelengths shorter than about $1.4 \mu \mathrm{m}$ (see Fig. 3b in $\mathrm{Fu}$ 1996).

\section{New parameterization of the asymmetry factor}

To provide a reliable parameterization of the asymmetry factor of cirrus clouds, we use 28 ice crystal size distributions based on in situ aircraft observations compiled by $\mathrm{Fu}$ (1996). For light scattering calculations, the size distributions are discretized into 30 bins ranging from 8.75 to $3100 \mu \mathrm{m}$ to resolve the structure of the size spectra. For hexagonal columns, the relationship between $L$ and $D$ follows Eq. (2.1) of Fu (1996). We thus have the mean effective aspect ratios for the 28 size distributions ranging from 0.34 to 0.99 , ice water content from $5.5 \times 10^{-4}$ to $0.28 \mathrm{~g} \mathrm{~m}^{-3}$, and $D_{\mathrm{ge}}$ from
18.6 to $139.2 \mu \mathrm{m}$. For hexagonal plates, we use the relationship between $L$ and $D$ following Pruppachar and Klett (1997), given by $L=1.7915 D^{0.474}$. The resulting ARs are from 3.14 to 16.68 , ice water content from $2.4 \times 10^{-4}$ to $0.28 \mathrm{~g} \mathrm{~m}^{-3}$, and $D_{\text {ge }}$ from 11.6 to $77.4 \mu \mathrm{m}$.

The single-scattering properties of hexagonal ice crystals are calculated using a geometric ray-tracing program (Takano and Liou 1989; Yang and Liou 1995). The ice crystals are assumed to be randomly oriented in space. We use the refractive indices of ice following $\mathrm{Fu}$ (1996). The single-scattering calculations are performed at 200 wavelengths. The solar spectra are divided into six bands (Fu 1996) and the data are weighted with the solar irradiance to obtain average values in each spectral band. The single-scattering properties for cirrus clouds containing hexagonal columns are the same as in Fu (1996) and we only need to calculate those for hexagonal plates. Following Fu (1996), the contribution of delta-function transmission through parallel planes at the zero-scattering angle is derived based on the conventional ray-tracing calculations (Takano and Liou 1989). Note that using the improved ray-tracing technique we cannot separate the contributions of the delta transmission as well as diffracted rays, but such separations are important in the radiative flux transfer calculations involving cirrus clouds (Fu 1996).

The asymmetry factor in the visible ranges from 0.766 to 0.836 for the 28 cirrus clouds containing ice columns and from 0.833 to 0.938 for ice plates. We derive asymmetry factors for cirrus clouds containing ice particles with rough surfaces, $g_{r}$, using $g_{r}=\left(g_{s}-\right.$ $\left.f_{d}\right) /\left(1-f_{d}\right)$, where $g_{s}$ is the asymmetry factor for ice particles with smooth surfaces and $f_{d}$ is the deltatransmission fraction. The $g_{r}$ ranges from 0.734 to 0.806 for the 28 cirrus clouds containing ice columns and from 0.783 to 0.898 for ice plates. After obtaining $g_{s}$ and $g_{r}$ along with the single-scattering albedo for given ice particle size distributions, we can derive $g_{s}^{\prime}$ and $g_{r}^{\prime}$ using Eq. (2.2).

We parameterize $g^{\prime}$ in terms of AR in the forms

$$
\begin{aligned}
& g_{s}^{\prime}=c_{s 0}+c_{s 1} \mathrm{AR}+c_{s 2} \mathrm{AR}^{2}, \\
& g_{r}^{\prime}=c_{r 0}+c_{r 1} \mathrm{AR}+c_{r 2} \mathrm{AR}^{2},
\end{aligned}
$$

for $0.1 \leq \mathrm{AR} \leq 1.0$ and

$$
\begin{aligned}
& g_{s}^{\prime}=p_{s 0}+p_{s 1} \ln (\mathrm{AR})+p_{s 2} \ln ^{2}(\mathrm{AR}), \\
& g_{r}^{\prime}=p_{r 0}+p_{r 1} \ln (\mathrm{AR})+p_{r 2} \ln ^{2}(\mathrm{AR})
\end{aligned}
$$


Table 1. Values of coefficients in Eqs. (3.1), (3.2), and (3.5) for aspect ratios between 0.1 and 1.0: units for $D_{\text {ge }}$ are in $\mu \mathrm{m}$.

\begin{tabular}{cccc}
\hline \hline Band limit $(\mu \mathrm{m})$ & $c_{s 2}$ & $c_{s 1}$ & $c_{s 0}$ \\
\hline $0.25-0.7$ & $1.349959 \times 10^{-1}$ & $-3.987320 \times 10^{-1}$ & $7.938904 \times 10^{-1}$ \\
$0.70-1.41$ & $1.115697 \times 10^{-1}$ & $-3.723287 \times 10^{-1}$ & $8.030084 \times 10^{-1}$ \\
$1.41-1.90$ & $9.853958 \times 10^{-2}$ & $-3.924784 \times 10^{-1}$ & $8.692241 \times 10^{-1}$ \\
$1.90-2.50$ & $5.557793 \times 10^{-2}$ & $-3.259404 \times 10^{-1}$ & $7.085850 \times 10^{-1}$ \\
$2.50-3.50$ & $-1.233493 \times 10^{-1}$ & $4.429054 \times 10^{-2}$ & $6.412701 \times 10^{-1}$ \\
$3.50-4.00$ & 0.0 & $-1.726586 \times 10^{-1}$ & \\
\hline & $d_{c 0}$ & $d_{c 1}$ & \\
$0.25-0.7$ & 1.0 & 0.0 & \\
$0.7-1.41$ & $9.843939 \times 10^{-1}$ & 0.0 & \\
$1.41-1.90$ & $9.551336 \times 10^{-1}$ & $-1.212890 \times 10^{-3}$ & \\
$1.90-2.50$ & $9.054886 \times 10^{-1}$ & $-2.444755 \times 10^{-3}$ & \\
$2.50-3.50$ & $4.480282 \times 10^{-1}$ & $-1.695611 \times 10^{-2}$ & \\
$3.50-4.00$ & $9.135134 \times 10^{-1}$ & $c_{r 1}$ & $7.386498 \times 10^{-1}$ \\
& $c_{r 2}$ & $-4.157560 \times 10^{-1}$ & $139 \times 10^{-1}$ \\
$0.25-0.7$ & $1.425497 \times 10^{-1}$ & $-3.873595 \times 10^{-1}$ & $8.230952 \times 10^{-1}$ \\
$0.7-1.41$ & $1.164948 \times 10^{-1}$ & $-4.302394 \times 10^{-1}$ & $8.529342 \times 10^{-1}$ \\
$1.41-1.90$ & $1.077919 \times 10^{-1}$ & $-3.608777 \times 10^{-1}$ & $6.967419 \times 10^{-1}$ \\
$1.90-2.50$ & $5.926338 \times 10^{-2}$ & $4.670750 \times 10^{-2}$ & $6.609769 \times 10^{-1}$ \\
$2.50-3.50$ & $-1.340814 \times 10^{-1}$ & $-2.408338 \times 10^{-1}$ &
\end{tabular}

for $1.0<\mathrm{AR} \leq 20$, where the coefficients are obtained by numerical fitting to the data for the 28 cirrus clouds containing ice columns and plates, respectively. Thus, for given $\mathrm{AR}$ and $D_{\text {ge }}$, we can derive the asymmetry factor using Eqs. (3.1)-(3.4) and Eq. (2.2) where $\tilde{\omega}$ is parameterized in terms of $D_{\text {ge }}$ from Fu (1996). Note that the obtained asymmetry factor is independent of $D_{\text {ge }}$ for wavelengths shorter than $1.4 \mu \mathrm{m}$.

We parameterize the delta-transmission fraction, $f_{d}$, in the following form:

$$
\begin{aligned}
f_{d}= & d_{c 0}\left(0.04844 \mathrm{AR}^{2}-0.1157 \mathrm{AR}\right. \\
& +0.1887) \exp \left(d_{c 1} D_{\mathrm{ge}}\right),
\end{aligned}
$$

for $0.1 \leq \mathrm{AR} \leq 1.0$ and

$$
\begin{aligned}
f_{d}= & d_{p 0}\left[-0.000165 \ln ^{2}(\mathrm{AR})+0.0974 \ln (\mathrm{AR})\right. \\
& +0.106] \exp \left(d_{p 1} D_{\mathrm{ge}}\right],
\end{aligned}
$$

for $1.0<\mathrm{AR} \leq 20$, where the coefficients are again obtained by numerical fitting to the data for the 28 cirrus clouds. The coefficients in Eqs. (3.1)-(3.6) are provided in Tables 1 and 2 for $0.1 \leq \mathrm{AR} \leq 1.0$ and $1.0<\mathrm{AR} \leq 20$, respectively. Note that $d_{c 1}$ and $d_{p 1}$ are zero for the bands $0.2-0.7$ and $0.7-1.41 \mu \mathrm{m}$.

Comparisons of the parameterizations and the reference calculations are shown for $g_{s}$ and $g_{r}$ versus AR in Fig. 4 and for $f_{d}$ versus AR in Fig. 5, in the band of 0.2-0.7 $\mu \mathrm{m}$, where $g$ and $f_{d}$ are only functions of AR. The agreements are similar for the other five bands.
Figure 6 shows $g_{s}, g_{r}$, and $f_{d}$ from parameterizations versus those from reference calculations for 28 ice particle size distributions using both columns and plates for all six bands in the solar radiation. The parameterization in terms of AR along with $D_{\text {ge }}$ well represents the variation of asymmetry factor and the deltatransmission fraction, with an averaged absolute relative error of about $0.4 \%$ for $g_{s}$ and $g_{r}$ and $3 \%$ for $f_{d}$. The parameterizations can be used for AR ranging from 0.1 to 20 .

In the radiative transfer calculations, the fraction of scattered energy residing in the forward peak, $f$, needs to be removed from the scattering parameters to incorporate the strong forward peak contribution in multiple scattering. We use

$$
f=\frac{1}{2 \tilde{\omega}}+f_{d}
$$

for ice particles with smooth surfaces (Fu 1996) but

$$
f=\frac{1}{2 \tilde{\omega}}
$$

for ice particles with rough surfaces. The singlescattering properties are then adjusted following the similarity principle for radiative transfer calculations (e.g., Liou 2002).

\section{Test of new parameterizations}

The discussion in section 2 indicates that the parameterization of asymmetry factor in terms of AR devel- 
TABLE 2. Values of coefficients for Eqs. (3.3), (3.4), and (3.6) for aspect ratios between 1.0 and 20: units for $D_{\text {ge }}$ are in $\mu \mathrm{m}$.

\begin{tabular}{cccc}
\hline \hline Band limit $(\mu \mathrm{m})$ & $p_{s 2}$ & $p_{s 1}$ & $p_{s 0}$ \\
\hline $0.25-0.70$ & $3.165543 \times 10^{-3}$ & $1.140557 \times 10^{-1}$ & $5.292852 \times 10^{-1}$ \\
$0.70-1.41$ & $2.014810 \times 10^{-3}$ & $1.143152 \times 10^{-1}$ & $5.425909 \times 10^{-1}$ \\
$1.41-1.90$ & $1.780838 \times 10^{-3}$ & $1.143814 \times 10^{-1}$ & $5.601598 \times 10^{-1}$ \\
$1.90-2.50$ & $6.987734 \times 10^{-4}$ & $1.071238 \times 10^{-1}$ & $6.023407 \times 10^{-1}$ \\
$2.50-3.50$ & $-1.882932 \times 10^{-2}$ & $1.353873 \times 10^{-1}$ & $6.473899 \times 10^{-1}$ \\
$3.50-4.00$ & $-2.277872 \times 10^{-2}$ & $1.914431 \times 10^{-1}$ & $4.634944 \times 10^{-1}$ \\
\hline & $d_{p 0}$ & $d_{p 1}$ & \\
\hline $.25-0.70$ & 1.0 & 0.0 & \\
$0.70-1.41$ & 1.0 & 0.0 & \\
$1.41-1.90$ & 1.0 & $-6.735732 \times 10^{-4}$ & \\
$1.90-2.50$ & $9.546090 \times 10^{-1}$ & $-2.739150 \times 10^{-3}$ & \\
$2.50-3.50$ & $5.298833 \times 10^{-1}$ & $-1.126258 \times 10^{-2}$ & \\
$3.50-4.00$ & $9.625839 \times 10^{-1}$ & $p_{r 1}$ & $4.653916 \times 10^{-1}$ \\
& $p_{r 2}$ & $7.070839 \times 10^{-2}$ & $4.816343 \times 10^{-1}$ \\
$0.25-0.70$ & $1.659297 \times 10^{-2}$ & $7.292324 \times 10^{-2}$ & $5.044055 \times 10^{-1}$ \\
$0.70-1.41$ & $1.478288 \times 10^{-2}$ & $7.367233 \times 10^{-2}$ & $5.555767 \times 10^{-1}$ \\
$1.41-1.90$ & $1.549338 \times 10^{-2}$ & $7.020179 \times 10^{-2}$ & $6.281019 \times 10^{-1}$ \\
$1.90-2.50$ & $1.351337 \times 10^{-2}$ & $1.246242 \times 10^{-1}$ & $4.098578 \times 10^{-1}$ \\
\hline $3.50-3.50$ & $-1.541111 \times 10^{-2}$ & $1.531992 \times 10^{-1}$ & \\
\hline
\end{tabular}

oped in section 3 can be generally applied to nonspherical ice particles with more complicated shapes. Herein we test this parameterization for fractal particles (Macke et al. 1996), aggregates, and spatial bullet rosettes (Yang et al. 2000), and with observations (Garrett et al. 2001), focusing on the visible radiation.

Considering randomized Koch fractals for irregular shaped ice particles, Macke et al. (1996) obtained an asymmetry factor of 0.74 . Such particles have rough surfaces and their individual components have an aspect ratio of about one.

Yang et al. (2000) constructed the aggregates using eight hexagonal elements with different given aspect ratios (Yang and Liou 1998). In their numerical computation, the number of hexagonal elements of which an aggregate consists is randomly selected from two to eight to average the effect of aggregate orientations. The selected hexagonal elements are attached together
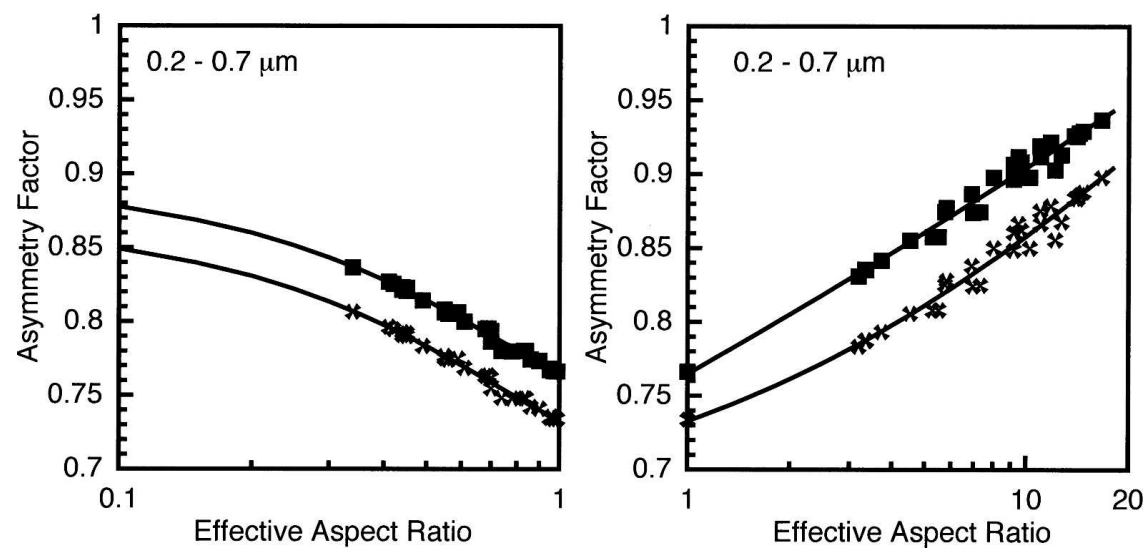

FIG. 4. Asymmetry factor vs the mean effective AR in the spectral interval $0.2-0.7 \mu \mathrm{m}$ for (left) AR smaller than 1 and (right) AR larger than 1 . The square and $\times$ symbols in each panel represent reference results from the geometric ray-tracing calculations using 28 measured ice crystal size distributions for ice particles with smooth and rough surfaces, respectively. The curves are from the parameterizations developed in this study. 

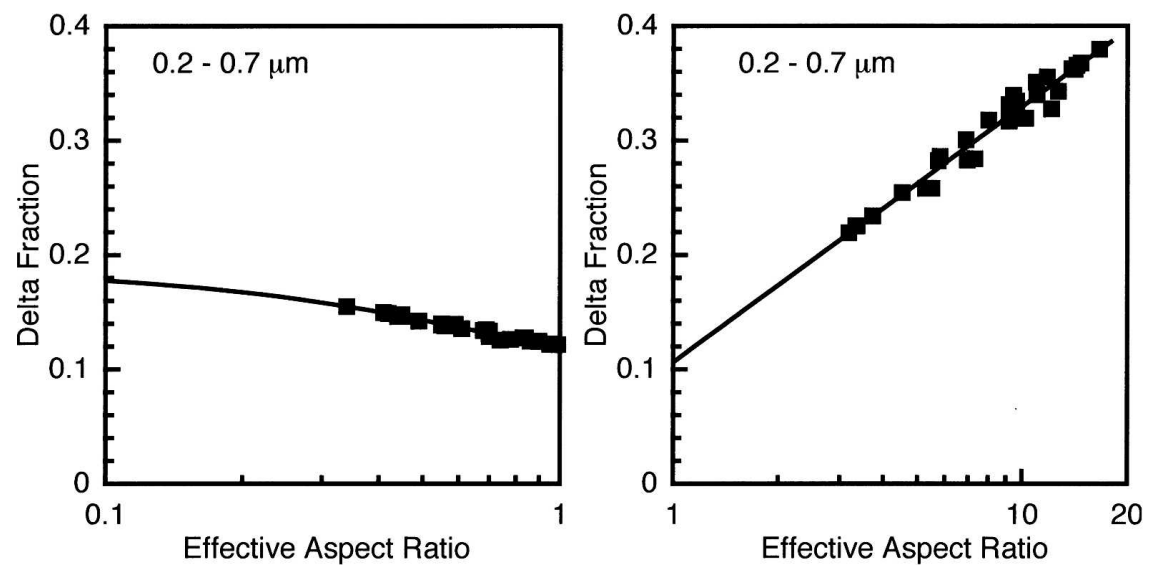

FIG. 5. As in Fig. 2, but for the forward delta-transmisson fraction of ice particles with smooth surfaces.

without overlap. The mean effective aspect ratio is 0.67 for their aggregates. Yang et al. (2000) considered the effect of rough surface by using a two-dimensional Gaussian probability function. They obtained an asymmetry factor of 0.76 .

Figure 7 shows the comparison of asymmetry factors of fractal ice particles and aggregates with those for ice particles with rough surfaces from our parameterization. The differences are smaller than 0.01. Such an agreement justifies our simple representation of ice particle surface roughness for its effect on the asymmetry factor.

Yang et al. (2000) also gave the asymmetry factor for spatial bullet rosettes composed of multiple (randomly selected from two to six) bullet branches with smooth surfaces (Yang and Liou 1998). The typical values of their asymmetry factors are also compared with our parameterizations in Fig. 7, which shows differences of less than about 0.01 .
Garrett et al. (2001) analyzed direct airborne measurements of the asymmetry factor of cirrus clouds. With and without delta-function transmission, the derived values of $g$ were $0.76 \pm 0.03$ and $0.74 \pm 0.03$, respectively. The majority of ice particles from the Cloud Particle Imager (CPI) were bullet rosettes (the average number of branches were six) and the mean aspect ratio was 0.4 (Garrett et al. 2001). The $g_{s}$ and $g_{r}$ for an AR of 0.4 from our parameterizations are 0.828 and 0.797 , which are significantly larger than those from observations. [The asymmetry factor of the bullet rosette with six branches and an aspect ratio of 0.4 is 0.837 based on the geometric ray-tracing method from Iaquinta et al. (1995).] It should be noted that the mean aspect ratio in Garrett et al. (2001) was underestimated since the CPI missed small ice particles. In the cases described in Garrett et al. these small crystals were responsible for $\sim 38 \%$ of the extinction coefficient. Assuming a mean effective aspect ratio of 1.0 for small ice
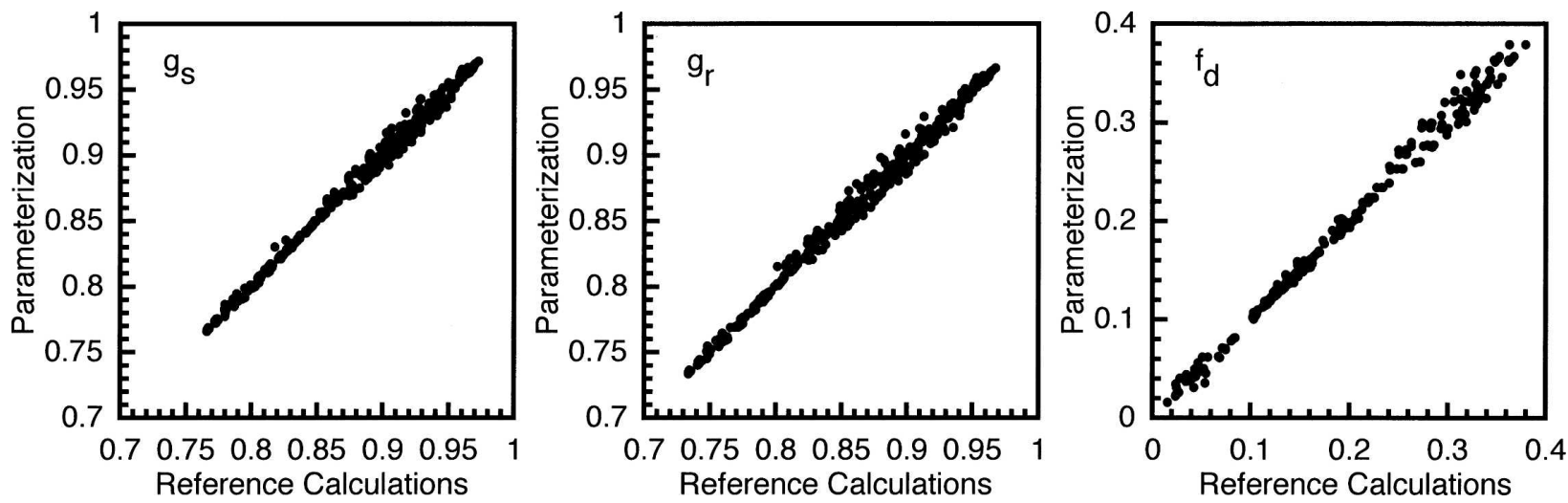

FIG. 6. Parameterization vs reference calculations for asymmetry factors for ice particles with smooth surfaces $\left(g_{s}\right)$, asymmetry factors for rough surfaces $\left(g_{r}\right)$, and the delta-transmission fraction $\left(f_{d}\right)$. Each panel contains 336 points for 28 ice particle size distributions with both ice columns and plates for all six bands in the solar radiation. 


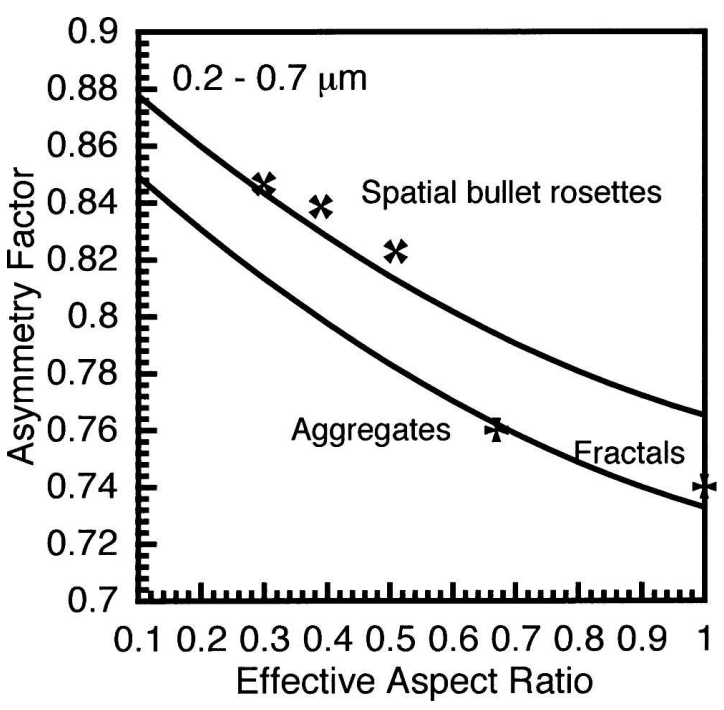

FIG. 7. Comparison of asymmetry factors based on the parameterization with those of spatial bullet rosettes with smooth surfaces, aggregates with rough surfaces, and fractal ice crystals in the visible.

particles, the AR with the consideration of small ice particles should be 0.63 (i.e., $1.0 \times 0.38+0.4 \times 0.62$ ). Thus, we have a $g_{s}$ of 0.798 and a $g_{r}$ of 0.766 , which agree with observed asymmetry factors within observational uncertainty.

It can be concluded that the parameterization of the asymmetry factor from this study along with the parameterizations of the extinction coefficient and singlescattering albedo from $\mathrm{Fu}$ (1996) can be generally applied to cirrus clouds containing various nonspherical ice particles. New research efforts need to be initiated in theoretical and modeling and observational studies to parameterize/diagnose AR for climate models. The observational studies require both in situ observational data analysis and the development of satellite remote sensing of ice crystal shape information in terms of AR that is not a parameter currently available from operational satellite retrieval products.

\section{More discussions on the new parameterization}

The asymmetry factor parameterization developed in this study is more general than previous parameterizations because of the following aspects. First, the new parameterization is generally valid for solid hexagonal ice particles including columns, plates, and their assemblages. Second, the new parameterization reproduces the asymmetry factor of nonhexagonal fractal crystals (Macke et al. 1996), which has also been widely used in climate and remote sensing studies. Third, the new parameterization can reproduce various previous param- eterizations (e.g., those shown in Fig. 1) by considering the assumed $\mathrm{AR}-D_{\mathrm{ge}}$ relationship and surface roughness. In addition, the new parameterization agrees well with in situ observations.

However, it should be noted that we did not consider the hollow ice crystals that may have different asymmetry factors for a given aspect ratio (Takano and Liou 1995; Yang et al. 2000; Schmitt et al. 2006). Schmitt et al. showed that for a given hollowness of 0.5 the asymmetry factors of bullet rosettes range from $\sim 0.79$ to $\sim 0.86$ due to the aspect ratio changes, while for a given aspect ratio of 0.5 the asymmetry factors are from $\sim 0.79$ to $\sim 0.83$ due to the change of hollowness. The impact of hollow ice crystals on cirrus asymmetry factor also depends on their percentage in total ice particles. Further research effort is required to consider such impact in the asymmetry factor parameterization.

It should also be noted that the ice particle surface roughness is another independent parameter to determine the asymmetry factor (Macke et al. 1996; Yang and Liou 1998). We have developed the new parameterization separately for ice particles with smooth and rough surfaces. Our simple consideration of ice particle surface roughness is justified by comparing the asymmetry factors of ice particles with rough surfaces recommended in Macke et al. (1996) and Yang et al. (2000).

There are several advantages for incorporating our new parameterization of the cirrus asymmetry factor in climate models over those shown in Fig. 1. First, the new parameterization is more general and physically based. Second, the new parameterization can go back to various previous parameterizations by specifying an assumed $\mathrm{AR}-D_{\mathrm{ge}}$ relationship [e.g., $\mathrm{AR}=1.236-$ $1.526 \times 10^{-2} D_{\mathrm{ge}}+1.004 \times 10^{-4} D_{\mathrm{ge}}^{2}-2.750 \times 10^{-7} D_{\mathrm{ge}}^{3}$ from $\mathrm{Fu}$ (1996) and $\mathrm{AR}=0.67$ with rough surfaces from Edwards et al. (2007)]. (Edwards et al. showed that the differences in cirrus reflected solar radiation using different cirrus radiative properties parameterization are mainly due to the differences in the asymmetry factor parameterization.) Third, the AR is a critically important parameter to consistently quantify the cirrus radiative forcing since it only has impact on the cirrus shortwave radiative forcing but has little impact on the longwave forcing (different from $D_{\text {ge }}$ and IWC that have an impact on both). (Note that cirrus longwave radiative forcing is dominated by the absorption and emission processes.)

\section{Application of the new parameterization to the cirrus radiative energy budget}

By incorporating the $g$ parameterization in terms of $\mathrm{AR}$ and $D_{\mathrm{ge}}$ into the $\mathrm{Fu}-\mathrm{Liou}$ radiation model (Fu and 

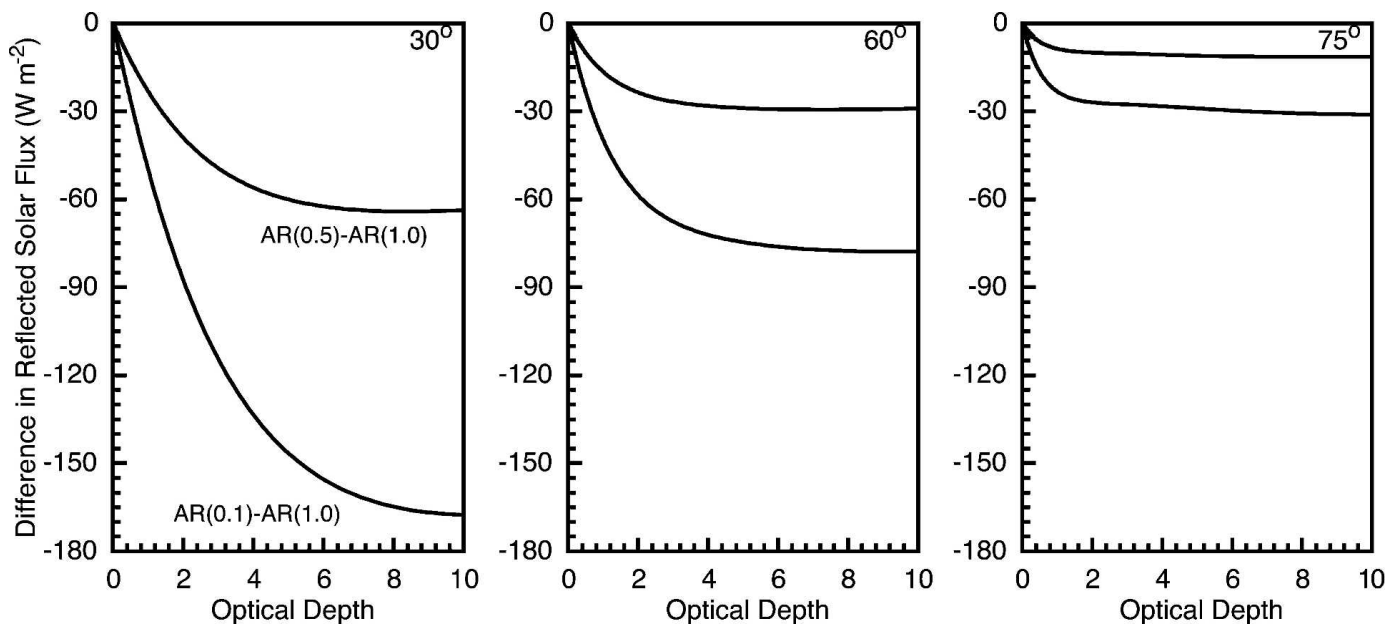

FIG. 8. Differences in reflected solar fluxes due to changes in the mean effective aspect ratio from 1.0 to 0.1 and from 1.0 to 0.5 for cirrus clouds containing ice particles with smooth surfaces. The solar zenith angles of $30^{\circ}, 60^{\circ}$, and $75^{\circ}$ are used in the calculations. The cirrus cloud is inserted between 10 and $12 \mathrm{~km}$ with a mean effective size of $45 \mu \mathrm{m}$.

Liou 1992, 1993; Fu 1996), this section presents the impact of ice particle mean aspect ratio and surface roughness on the radiative energy balance through their effects on the symmetry factor. We will also compare results using the new parameterization with those based on $g$ from $\mathrm{Fu}$ (1996).

The U.S. Standard Atmosphere, 1976 profiles (COESA 1976) are used in the solar radiative transfer calculations. We assume a surface albedo of 0.1 and the solar constant of $1365 \mathrm{~W} \mathrm{~m}^{-2}$. A cirrus cloud is inserted between 10 and $12 \mathrm{~km}$ with a $D_{\text {ge }}$ of $45 \mu \mathrm{m}$. We vary the cirrus optical depth from 0 to 10 by changing the ice water content. Figure 8 shows the effect of AR on the reflected solar radiation as a function of cirrus optical depth for solar zenith angles of $30^{\circ}, 60^{\circ}$, and $75^{\circ}$. We can see that the magnitude of the differences increases with the increase of optical depth (within the range of optical depth considered) but decreases with the increase of solar zenith angles. For a solar zenith angle of $60^{\circ}$ and a cirrus optical depth of 4 , the differences in reflected solar radiation are about -30 or $-70 \mathrm{~W} \mathrm{~m}^{-2}$, respectively, due to changes in AR from 1.0 to 0.5 or from 1.0 to 0.1 . Figure 9 is similar to Fig. 8 but shows the effect of ice particle surface roughness. Such an
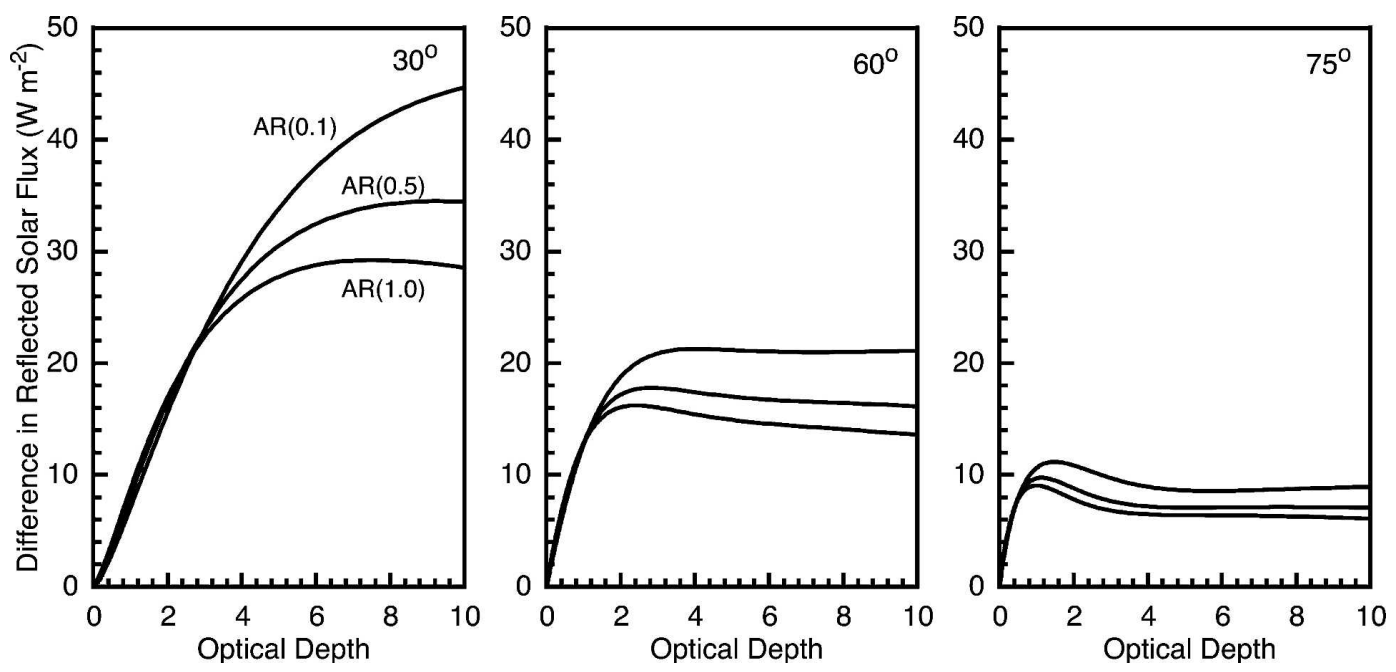

FIG. 9. Differences in reflected solar fluxes between cirrus clouds containing ice particles with rough and smooth surfaces for the solar zenith angles of $30^{\circ}, 60^{\circ}$, and $75^{\circ}$. The mean effective aspect ratios of $0.1,0.5$, and 1.0 are used in the calculations. The cirrus cloud is inserted between 10 and $12 \mathrm{~km}$ with a mean effective size of $45 \mu \mathrm{m}$. 


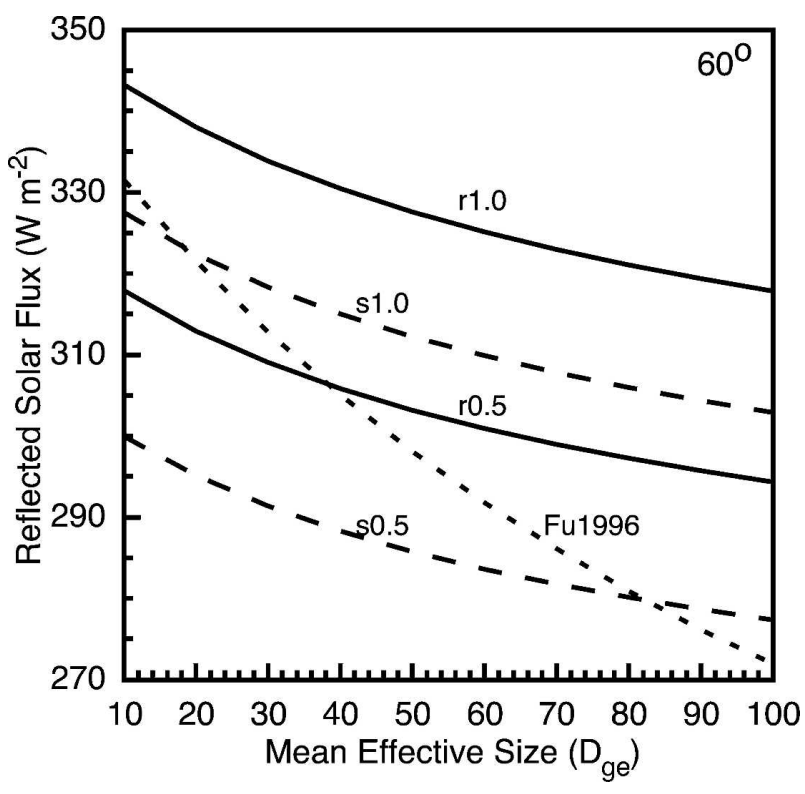

FIG. 10. Comparison of reflected solar fluxes between using the new $g$ parameterization (solid lines for ice particles with smooth surfaces and dashed lines for rough surfaces) and that from $\mathrm{Fu}$ (1996) (dotted lines). The mean effective aspect ratios of 0.5 and 1.0 are used in the new parameterization. The cirrus cloud is inserted between 10 and $12 \mathrm{~km}$ with an optical depth of 4 and a solar zenith angle of $60^{\circ}$.

effect is larger for smaller AR. For a solar zenith angle of $60^{\circ}$ and a cirrus optical depth of 4 , the differences in reflected solar radiation due to the consideration of ice particle surface roughness are from 15 to $21 \mathrm{~W} \mathrm{~m}^{-2}$, depending on the AR used.

For the comparison of the $\mathrm{Fu}$ (1996) $g$ parameterization in terms of $D_{\text {ge }}$ with $g$ in terms of AR and $D_{\text {ge }}$, we still consider the cirrus cloud between 10 and $12 \mathrm{~km}$ but with a fixed optical depth of 4 . A solar zenith angle of $60^{\circ}$ is used. We change the $D_{\text {ge }}$ from 10 to $100 \mu \mathrm{m}$ (the ice water content is also changed to keep the optical depth constant). For the new parameterization we use the ARs of 0.5 and 1.0 for ice particles with both smooth and rough surfaces. The reflected solar fluxes are shown in Fig. 10 as functions of mean effective size. For given AR the reflected solar fluxes decrease with the increase of $D_{\mathrm{ge}}$. This is because the coalbedo, $1-\tilde{\omega}$, increases with $D_{\text {ge }}$, which enhances cloud absorption (for given optical depth), and increases $g$ in the bands with significant absorption. Both processes contribute to the decrease of reflected solar fluxes but the former dominates (the impact of $D_{\text {ge }}$ through $g$ for given AR is only about $3 \mathrm{~W} \mathrm{~m}^{-2}$ based on our sensitivity calculations). The large decrease of the reflected fluxes with $D_{\text {ge }}$ using Fu (1996) is mainly due to the increase of $g$ with $D_{\text {ge }}$ (Fig. 1). The results of Fu are about between those using ARs of 0.5 and 1.0 for ice particles with smooth surfaces. However, there are two issues in the $g$ parameterization of Fu (1996). First, the dependence of $g$ on $D_{\text {ge }}$ is not generic but depends on the assumed relationship between the aspect ratio and ice particle size built into the parameterization. This shortcoming applies to all parameterizations shown in Fig. 1. Second, it does not consider the effect of ice particle surface roughness on the light scattering, which seems to be a common feature of natural ice particles in cirrus clouds (e.g., Macke et al. 1996; Yang and Liou 1998; Garrett et al. 2001; Baran and Francis 2004; Edwards et al. 2007). We recommend that the parameterization of $g$ in terms of $\mathrm{AR}$ and $D_{\text {ge }}$ for ice particles with rough surfaces should be used in the global climate models.

\section{Summary and conclusions}

We have identified the aspect ratio of a nonspherical ice particle as the key microphysical parameter to determine its asymmetry factor for solar radiation. We define the mean effective aspect ratio (AR) for cirrus clouds containing various nonspherical ice particles. We find that for wavelengths longer than $1.4 \mu \mathrm{m}$ where the absorption becomes significant, the asymmetry factor also depends on ice particle size. This is largely because the change of single-scattering albedo modifies the relative contribution of diffracted rays to the scattered light.

We have developed a new parameterization of asymmetry factor of cirrus clouds in terms of AR and $D_{\text {ge }}$ for solar radiation based on geometric ray-tracing calculations for hexagonal ice crystals with a simple representation of particle surface roughness. Twenty-eight ice crystal size distributions based on in situ aircraft observations are used for both columns and plates, with the AR ranging from about 0.3 to 17 . The new parameterization is independent of $D_{\mathrm{ge}}$ for wavelengths shorter than $1.4 \mu \mathrm{m}$.

We show that the new parameterization reproduces the asymmetry factors of complicated ice particles such as spatial bullet rosettes, aggregates with rough surfaces, and fractal crystals and agrees well with observations. We have thus provided a more generalized parameterization of the asymmetry factor for nonspherical ice particles that can be properly applied to cirrus clouds containing various nonspherical ice particles.

The radiative transfer calculations are performed to test the new parameterization of the asymmetry factor. We show that, for a cirrus cloud with an optical depth of 4 and a solar zenith angle of $60^{\circ}$, changes in AR from 1.0 to 0.5 or from 1.0 to 0.1 result in differences in reflected solar fluxes of about -30 or $-70 \mathrm{~W} \mathrm{~m} \mathrm{~m}^{-2}$, 
respectively. For the same cloudy conditions, the effect of ice particle surface roughness on the reflected solar flux is found to be about $20 \mathrm{~W} \mathrm{~m}^{-2}$.

Acknowledgments. The author wishes to thank Prof. T.C. Grenfell for his comments and suggestions on the manuscript. This work was supported by NASA Grants NNG04GM23G and NNG05GA19G, a Boeing contract, and LANL IGPP Award.

\section{REFERENCES}

Baran, A. J., and P. N. Francis, 2004: On the radiative properties of cirrus cloud at solar and thermal wavelengths: A test of model consistency using high-resolution airborne radiance. Quart. J. Roy. Meteor. Soc., 130, 763-778.

Chou, M. D., K. T. Lee, and P. Yang, 2002: Parameterization of shortwave cloud optical properties for a mixture of ice particle habits for use in atmospheric models. J. Geophys. Res., 107, 4600, doi:10.1029/2002JD002061.

COESA, 1976: U.S. Standard Atmosphere, 1976. NOAA, 227 pp.

Ebert, E. E., and J. A. Curry, 1992: A parameterization of ice cloud optical properties for climate models. J. Geophys. Res., 97, 3831-3836.

Edwards, J. M., S. Havemann, J. C. Thelen, and A. J. Baran, 2007: A new parameterization for the radiative properties of ice crystals: Comparison with existing schemes and impact in a GCM. Atmos. Res., 83, 19-34.

Francis, P. N., A. Jones, R. W. Saunders, K. P. Shine, A. Slingo, and Z. Sun, 1994: An observational and theoretical study of the radiative properties of cirrus: Some results from ICE'89. Quart. J. Roy. Meteor. Soc., 120, 809-848.

Fu, Q., 1996: An accurate parameterization of the solar radiative properties of cirrus clouds for climate models. J. Climate, 9, 2058-2082.

— , and K. N. Liou, 1992: On the correlated $k$-distribution method for radiative transfer in nonhomogeneous atmospheres. J. Atmos. Sci., 49, 2139-2156.

— , and - 1993: Parameterization of the radiative properties of cirrus clouds. J. Atmos. Sci., 50, 2008-2025.

Garrett, T. J., P. V. Hobbs, and H. Gerber, 2001: Shortwave, single-scattering properties of arctic ice clouds. J. Geophys. Res., 106, $15155-15172$.

Grenfell, T. C., and S. G. Warren, 1999: Representation of a nonspherical ice particle by a collection of independent spheres for scattering and absorption of radiation. J. Geophys. Res., 104, 31 697-31 709.

Iaquinta, J., H. Isaka, and P. Personne, 1995: Scattering phase function of bullet rosette ice crystals. J. Atmos. Sci., 52, 14011413.

Liou, K. N., 2002: An Introduction to Atmospheric Radiation. 2d ed. Academic Press, 583 pp.

Macke, A., 1993: Scattering of light by polyhedral ice crystals. Appl. Opt., 32, 2780-2788.

_ J. Muller, and E. Rascke, 1996: Single scattering properties of atmospheric crystals. J. Atmos. Sci., 53, 2813-2825.

McFarquhar, G. M., P. Yang, A. Macke, and A. J. Baran, 2002: A new parameterization of single scattering solar radiative properties for tropical anvils using observed ice crystal size and shape distributions. J. Atmos. Sci., 59, 2458-2478.

Pruppacher, H. R., and J. D. Klett, 1997: Microphysics of Clouds and Precipitation. $2 \mathrm{~d}$ ed. Kluwer Academic, $954 \mathrm{pp}$.

Schmitt, C. G., J. Iaquinta, and A. J. Heymsfield, 2006: The asymmetry parameter of cirrus clouds composed of hollow bullet rosette-shaped ice crystals from ray-tracing calculations. $J$. Appl. Meteor. Climatol., 45, 973-981.

Stephens, G. L., S.-C. Tsay, P. W. Stackhouse Jr., and P. J. Flatau, 1990: The relevance of the microphysical and radiative properties of cirrus clouds to climatic feedback. J. Atmos. Sci., 47, 1742-1753.

Takano, Y., and K. N. Liou, 1989: Solar radiative transfer in cirrus clouds. Part I: Single scattering and optical properties of hexagonal ice crystals. J. Atmos. Sci., 46, 3-19.

- and - 1995: Radiative transfer in cirrus clouds. Part III: Light scattering by irregular ice crystals. J. Atmos. Sci., 52, 818-837.

Yang, P., and K. N. Liou, 1995: Light scattering by hexagonal ice crystals: Comparison of FDTD and geometric optics models. J. Opt. Soc. Amer., 12, 162-176.

— , and — 1998: Single-scattering properties of complex ice crystals in terrestrial atmosphere. Contrib. Atmos. Phys., 71, 223-248.

- — - K. Wyser, and D. Mitchell, 2000: Parameterization of the scattering and absorption properties of individual ice crystals. J. Geophys. Res., 105, 4699-4718. 